\title{
What Do You Need to Know About GLP-1 Receptor Agonists?
}

Kim A. Carmichael, MD-Series Editor

Glucagon-like peptide 1 (GLP-1) agonists are an important class of medications for patients with type 2 diabetes and should be considered for long-term therapy. A study of noninsulin treatment of type 2 diabetes in the United States showed that there has been a gradual decline in metformin use (from $57.0 \%$ in 2015 to $46.0 \%$ in 2019), an increase of GLP-1 agonist monotherapy use (from 4.3\% in 2015 to $8.5 \%$ in 2019), and an increase in overall combined therapy use (from 9.8\% in 2015 to $18.2 \%$ in 2019). ${ }^{\text {Adequate management }}$ of diabetes with safe glycemic control is essential for reducing progression of chronic kidney disease, cardiovascular disease, and nonalcoholic fatty liver disease (NAFLD).

\section{Q. What are GLP-1 receptor agonists?}

A. GLP-1 was postulated to be involved with glucose homeostasis for many decades, but in the 1970s, scientists were able to use techniques such as radioimmunoassay and recombinant DNA approaches to better isolate the gene sequences and study GLP-1's biochemistry. ${ }^{2}$ By 1987 , GLP-1 was shown to stimulate insulin secretion, and in 1992, the first GLP-1 agonist (ie, exenatide) was isolated and extracted from Heloderma suspectum lizard venom. ${ }^{2}$ By 2005 , exenatide became commercially available as a twice-daily injection. In subsequent years, multiple formulations and analogs have been developed, including once daily injectable, once weekly injectable, and once daily oral medications.

GLP-1 agonists stimulate insulin secretion, inhibit glucagon secretion, decrease food intake, and slow gastric emptying, providing a multifactorial benefit for individuals with diabetes. ${ }^{2,3}$ They are recommended as first-line treatment options, with or without metformin, according to the Standards of Medical Care in Diabetes-2022 from the American Diabetes Association. ${ }^{4}$ They are preferred over insulin whenever possible. They are particularly beneficial for individuals with established atherosclerotic cardiovascular disease (ASCVD) or are at high risk for heart or kidney complications. Known

\section{AFFILIATIONS:}

John T. Milliken Department of Medicine, Division of Endocrinology, Metabolism, and Lipid Research, Washington University School of Medicine, St Louis, Missouri

\section{CITATION:}

Carmichael KA. What do you need to know about GLP-1 receptor agonists? Consultant. 2022;62(2):e39-e41. doi:10.25270/con.2022.02.00004

\section{DISCLOSURES:}

The authors report no relevant financial relationships.

\section{CORRESPONDENCE:}

Kim A. Carmichael, MD, Professor of Medicine, John T. Milliken Department of Medicine, Washington University School of Medicine, 660 S Euclid Ave, St Louis, MO 63110-1010 (carmichaelk@wustl.edu)

physiologic effects include reduction of inflammation and stimulation of crypt fission affecting intestinal growth factor, as well as direct effects on the cardiovascular system.

GLP-1 agonists promote weight reduction in individuals with diabetes; liraglutide and semaglutide are also approved by the US Food and Drug Administration (FDA) for weight management, even in the absence of diabetes. There are a few studies that also demonstrate potential benefit for nonalcoholic steatohepatitis (NASH), 2,5,6 Parkinson disease, and Alzheimer dementia. ${ }^{2}$

\section{Q. What are the indications for GLP-1 agonist use?}

A. There are currently multiple FDA-approved GLP-1 agonist formulations for use in the United States. Exenatide is indicated for individuals with type 2 diabetes as a twice daily or once weekly injection, although the weekly dose is more beneficial for weight management. ${ }^{2}$ Liraglutide and lixisenatide are administered via daily injection and are indicated for type 2 diabetes; liraglutide is also indicated for weight management. Dulaglutide is administered via weekly injection and is indicated for type 2 diabetes. Semaglutide is available as a weekly injection or daily pill for type 2 diabetes or weekly injection for weight management.

\section{Q. What are the potential cardiovascular benefits of GLP-1 agonists?}

A. Cardiovascular disease is the most common cause of death among individuals with diabetes. ${ }^{3}$ In addition to improving glycemic control, without significant 
risk of hypoglycemia (unless given with insulin or sulfonylurea therapy), GLP-1 agonists promote reductions in weight, systolic blood pressure, triglyceride levels, as well as total and low-density lipoprotein cholesterol levels. ${ }^{5}$ They may also have more direct benefits for cardiovascular physiology. Endogenous GLP-1 increases flow-mediated vasodilation, although synthetic degradation-resistant formulations do not. ${ }^{2}$ GLP-1 agonists increase heart rate and cardiac output, as well as reduce cardiac inflammation that may affect myocardial infarction size.

In a 2020 review of cardiovascular outcomes trials of GLP-1 agonists compared with placebo or other agents, all showed noninferiority of GLP-1 agonists in composite cardiovascular risk, and most showed beneficial outcomes. ${ }^{3}$ The Standards of Medical Care in Diabetes-2022 from the American Diabetes Association list dulaglutide, liraglutide, and injectable semaglutide as showing established benefit for ASCVD, with daily lixisenatide and weekly exenatide being neutral. ${ }^{4}$ The formal recommendations include adding GLP-1 agonist or sodium-glucose cotransporter 2 inhibitor therapy, when possible, for individuals with high cardiovascular risk.

In terms of patients with congestive heart failure, the American Diabetes Association's recommendations list GLP-1 agonists as having a neutral effect. However, a recent review suggests that there may be a greater benefit among individuals with heart failure with preserved ejection fraction compared with those with reduced ejection fraction. ${ }^{7}$

\section{Q. What are the microvascular outcomes for patients taking GLP-1 agonists?}

\section{A. According to a meta-analysis of} randomized controlled trials, including 60 studies and 60,077 patients, there was no change in the incidence of diabetic retinopathy, macular edema, retinal detachment, or retinal hemorrhage. ${ }^{8}$ However, there was a higher incidence of vitreous hemorrhage (among studies assessed as having low-quality evidence). There was also no relevant change in the estimated glomerular filtration rate, but there was a marginal reduction in the urinary albumin-to-creatinine ratio. ${ }^{8}$

In a Scandinavian cohort study involving 38,731 new users of GLP-1 agonists, there were significantly fewer renal events-including the need for renal replacement therapy, hospitalization for renal events, or death from renal causes-compared with users of dipeptidyl peptidase- 4 inhibitors. ${ }^{9}$

\section{Q. What are the benefits for NAFLD for patients taking GLP-1 agonists?}

A. NAFLD is present in about $70 \%$ of individuals with diabetes and has serious metabolic consequences, including cirrhosis. ${ }^{10}$ It is often underrecognized and is linked to poor diabetes control. ${ }^{11}$

In a meta-analysis of 8 randomized controlled trials, GLP-1 agonist therapy, compared with "standard of care," showed significant improvements with alanine aminotransferase, aspartate aminotransferase, gamma glutamyl transaminase, and glycosylated hemoglobin. ${ }^{6}$ There was reduction in liver fat content by imaging and histologic improvement in biopsy resolution.

In a mini review (ie, observational studies, randomized controlled trials, and meta-analyses) of GLP-1 agonists in individuals with NAFLD, the researchers called for greater awareness, screening, and strategies to promote weight loss (possibly including liraglutide and semaglutide). ${ }^{5}$ At the present time, there are no FDA-approved pharmacologic treatment options for NAFLD, but from this review, the benefits of GLP-1 agonists have been confirmed among individuals with biopsyconfirmed NASH.

Although hepatocytes do not have GLP-1 receptors, GLP-1 agonists have several indirect pathways to affect hepatic steatosis, largely related to peripheral and portal insulin and glucagon concentrations. ${ }^{5}$ These include hepatocyte mitochondrial function, hepatic insulin sensitivity, and abatement of adipose tissue lipotoxicity. Currently, limited studies are inconclusive to assess the benefit of GLP-1 agonists for treating hepatic fibrosis, but there are ongoing trials to look at long-term outcomes.

\section{Q. What are the potential} drawbacks of GLP-1 agonists?

A. The most common adverse effects of GLP-1 agonists include mild to moderate gastrointestinal symptoms (ie, nausea, vomiting, dyspepsia, and diarrhea), possible pancreatitis, and a slight increase in the risk of cholelithiasis. ${ }^{5}$ The potential human risk of medullary thyroid carcinoma is unknown but is listed as an FDA warning as a matter of precaution, particularly among individuals at risk for this uncommon form of cancer.

\section{Conclusions}

GLP-1 agonists are valuable agents for treating type 2 diabetes and may be used as first-line agents with or without metformin. They should be strongly considered for individuals with known or high risk for cardiovascular disease. GLP1 agonists are promising agents for the management of individuals with NAFLD. They have a favorable safety profile and are generally well-tolerated.

\section{References}

1. Heyward J, Christopher J, Sarkar S, Shin JI, Kalyani RR, Alexander GC. Ambulatory noninsulin treatment of type 2 diabetes mellitus in the United States, 2015 to 2019. Diabetes Obes Metab. 2021;23(8):1843-1850. https:// doi.org/10.1111/dom.14408

2. Drucker DJ, Habener JF, Holst JJ. Discovery, characterization, and clinical development of the glucagon-like peptides. J Clin Invest. 2017;127(12):4217-4227. https://doi. org/10.1172/jci97233

3. Sheahan KH, Wahlberg EA, Gilbert MP. An overview of GLP-1 agonists and recent cardiovascular outcomes trials. Postgrad Med J. 2020;96(1133):156-161. https://doi. org/10.1136/postgradmedj-2019-137186

4. American Diabetes Association Professional Practice Committee; American Diabetes 
Association Professional Practice Committee, Draznin B, et al. Pharmacologic approaches to glycemic treatment: standards of medical care in diabetes-2022. Diabetes Care. 2022;45(Supplement_1):S125-S143.

https://doi.org/10.2337/dc22-s009

5. Patel Chavez C, Cusi K, Kadiyala S. The emerging role of glucagon-like peptide-1 receptor agonists for the management of NAFLD. $J$ Clin Endocrinol Metab. 2022;107(1):29-38. https://doi.org/10.1210/clinem/dgab578

6. Ghosal S, Datta D, Sinha B. A meta-analysis of the effects of glucagon-like-peptide 1 receptor agonist (GLP1-RA) in nonalcoholic fatty liver disease (NAFLD) with type 2 diabetes (T2D). Sci Rep. 2021;11(1):22063. https://doi.org/10.1038/s41598-021-01663-y 7. Khan MS, Fonarow GC, McGuire DK, et al. Glucagon-like peptide 1 receptor agonists and heart failure: the need for further evidence generation and practice guidelines optimization. Circulation. 2020;142(12):12051218. https://doi.org/10.1161/circulationaha.120.045888

8. Avgerinos I, Karagiannis T, Malandris K, et al. Glucagon-like peptide-1 receptor agonists and microvascular outcomes in type 2 diabetes: A systematic review and meta-analysis. Diabetes Obes Metab. 2019;21(1):188193. https://doi.org/10.1111/dom.13484

9. Pasternak B, Wintzell V, Eliasson B, et al. Use of glucagon-like peptide 1 receptor agonists and risk of serious renal events: Scandinavian cohort study. Diabetes Care. 2020;43(6):1326-1335. https://doi. org $/ 10.2337 / \mathrm{dc} 19-2088$

10. Interview with Kim A. Carmichael, author of "What's the Significance of NAFLD in Diabetes?" Consultant360. December 20, 2017. Accessed January 13, 2022. https://www. consultant360.com/content/nafld-diabetes-what-primary-care-should-know

11. Alexopoulos AS, Duffy R, Kobe EA, et al. Underrecognition of nonalcoholic fatty liver disease in poorly controlled diabetes: a call to action in diabetes care. $J$ Endocr Soc. 2021;5(12):bvab155. https://doi.org/10.1210/ jendso/bvab155 